\title{
Convergence of DSRC and WiMAX Technology for Intelligent Transportation System
}

\author{
D. Kandar, C. Subathradevi, S. Porkodi, C. Auxilium Princy, M. Nithya, and E. Bhuvaneswari
}

\begin{abstract}
The geographical coverage area of communication is increased for the efficiency of the intelligent transportation system (ITS) customers. The maximum extent of coverage area provides the beneficiary of satisfying the customers with the desired needs of the customers, as it keeps changing rapidly. The noble technology WiMAX helps to achieve the desired goal for mobile users. This convergence of two Nobel techniques like DSRC and WiMAX system can be applied to transport infrastructure and vehicles for an efficient communication of the mobile units on the road.
\end{abstract}

Index Terms - Intelligent transportation system, wireless interoperability microwave access (WiMAX), dedicated shortrange communication (DSRC).

\section{INTRODUCTION}

During the twelve months to the last of Mar 09 in Australia, 249 people passed from 228 fatal road crashes. Fatal crashes maximum by 2.4 per cent by comparing with the previous twelve-month period and $8 \%$ per yr over the 3 years to Mar 09 [1] and [2].

The Monash University Accident Research Centre (MUARC) has established itself as the leading Australian organization undertaking research into the impact of in-vehicle Intelligent Transport Systems (ITS) on driver performance, behavior and safety [3]. Incidentally, India holds the dubious distinction of registering the highest number of road accidents in the world. According to the experts at the National Transportation Planning and Research Centre (NTPRC) the number of road accidents in India is three times higher than that prevailing in developed countries. The number of accidents for 1000 vehicles in India is as high as 35 while the figure ranges from 4 to 10 in developed countries [4]. In 2009, the death toll rose to 14 per hour as opposed to 13 the previous year in India. Due to road accidents the total number of deaths every year has now passed the 135,000 mark, according to the latest report of National Crime Records Bureau or NCRB [5]. Majority of road accidents are caused human error say senior police officials, according to a news report in the TOI today.

The greatest threat to our nations economic is posed by congestion. By Nationwide, drivers waste about four billion hours and over two billion gallons of fuel each year by sitting idle in traffic. It increases in emission levels and reduced quality of life. Businesses lose an estimated two hundred

Manuscript received September 4, 2012; revised December 18, 2012.

The authors are all with the SKP Engineering College, Thiruvannamalai, Tamilnadu, India (e-mail: kdebdatta@gmail.com). dollar billion per year due to traffic, which delays in delivery, and impacts just manufacturing. It is clear that, traffic increases when of traffic accidents are not present. So there are more benefits when using DSRC.Is improves safety level. Additionally, we have data collection for DSRC. The data can be changed as information and used as traffic management centers to modify route traffic for minimize the congested path. This will saves time, cost and minimize the emission levels. Electronic payment services allow vehicles to move through toll plazas without queuing. [6].

DSRC (IEEE 802.11p) complements other techniques such as WiFi, WiMAX and other communications protocols by providing the highest data rates for Intelligent Transportation System (ITS). As the increase for mobile users is growing with a positive plotted graph linearly ever, it is anticipated that in near future each and every car on the road would surely have a mobile unit for the purpose of communication. When this comes true, the communication among the mobile units causes high interferences and DSRC is not sufficient to tackle scenario in near future.

Motorola is the new entrant in the field of intelligent vehicle communication field and it wants to use Mobile WiMAX technology instead. WiMAX (Worldwide Interoperability for Microwave Access) technology which is based on IEEE 802.16 standards is one of the emerging wireless technology that provide us high speed mobile data and telecommunication services. WiMAX is Internet Protocol based wireless technology. WiMAX can provide broadband wireless access (BWA) up to 30 miles to 70 miles for fixed stations, and 3 - 10 miles for mobile stations.

It ensure that the broadband wireless technology manufactured for user to use interoperate from base station to base station. The advantage of WiMAX, it can provide efficient data transfer between number of users at a time using single base station with security. It has multi-functionality within the WiMAX, it can provide various aspects within a time. They are $3 \mathrm{G}$ and $4 \mathrm{G}$.

\section{WIMAX 2 TECHNOLOGY FOR MOBILE ENVIRONMENT}

Intel Samsung which is IEEE 802.16 standard promising to speed up WiMAX 2 competences. Wimax Forum is convinced that Wimax 2 will speedily enlarge the Mobile WiMAX. It is wireless telecommunication to speed up the process. WiMAX forum is working to speed up the outline for WiMAX 2 devices. According to Wimax Forum there are 620 million people using it globally. WiMAX has a various type of spectrum bands within $2.3 \mathrm{GHz}, 2.5 \mathrm{GHz}, 3.5 \mathrm{GHz}$ and 5.8 $\mathrm{GHz}$. 
In recent years, Mobile WiMax has received wide interest for next generation wireless communications [7], [8]. Two key technologies, Multiple Input Multiple Output (MIMO) and Orthogonal Frequency Division Multiplexing (OFDM) have been adopted in Mobile WiMAX standard [9], which enable high data rate transmission over multipath and fading channels. An ITS (Intelligent Transportation System) is to make the vehicles more intelligent in order to enhance the safety and at the same time increases the efficiency of highway utilization [10], is the deployment environment where the work will be implemented. WIMAX provides broadband wireless access up to 30 miles for fixed stations and $3-10$ miles for mobile stations [11].

\section{Wimax System for Data Transmission (See Fig. 1).}

The WiMAX system, displays the calculation of the Bit Error Rate (BER), with the number of error count and number of bits along with the compatible rate ID and the Estimation of SNR (Signal to Noise Ratio). It also displays the Spectrum scope of the transmitter to antenna respectively i.e. $(2 \times 1)$.

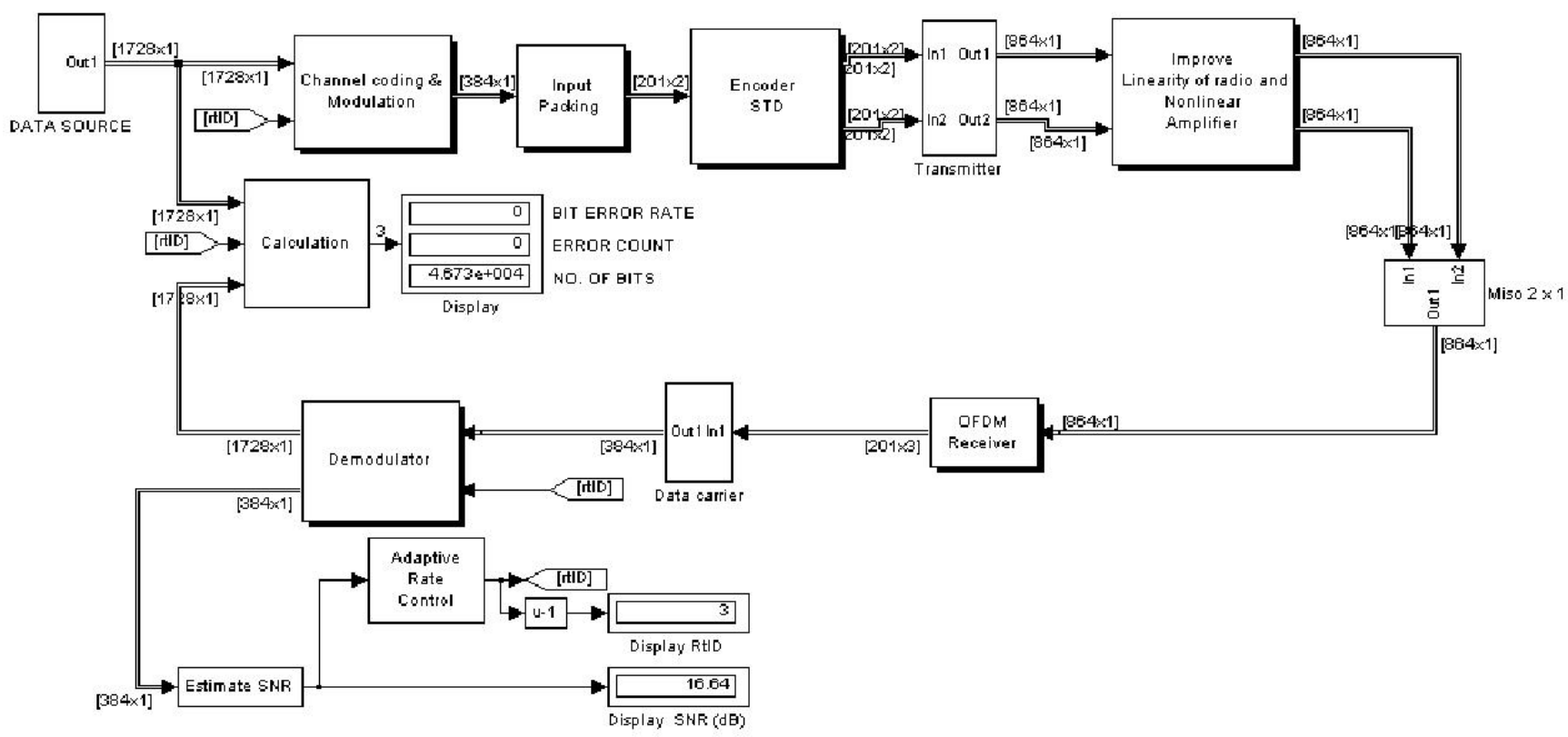

Fig. 1. WiMAX system-running simulation-screenshot.

\section{THE Mimo SySTEM FOR DATA COMMUNICATION}

The system comprises of developing the deployment of MIMO unit for exploring the data throughput and link range without additional bandwidth or increased transmit power. The system capacity can be significantly improved if multiple transmit and receive antennas are used to form MIMO channels [12]-[15]. The system proceeds with the deployment of WIMAX unit as it helps to cover the intended coverage area nearly a range of approximately the size of a city. WIMAX communications consist of management and data messages. Management messages are used to govern communications parameters, and data messages carry the data to be transmitted over wireless links [16]. This work actually takes control at this point of the system by the convergence of two units MIMO and WIMAX. The implementation of MIMO in WIMAX i.e. the MIMAX is done to improve the geographical coverage distance of communication.

The system description starts with the explication of the MIMO deployment unit proceeding with the WIMAX unit, convergence of both the WIMAX and MIMO unit and coming to the completion of the system with the description of Spread Spectrum based DSRC system.

\section{A. Multiple Input Multiple Output}

Deployment of the MIMO unit is carried as the initial step of the work to exploit the full spatial diversity order and employs symbol -wise Maximum Likelihood (ML) decoding. MIMO is a noble technology that can dramatically increase the spectral efficiency by using antenna arrays at both the transmitter and receiver [17].

The STBC (Space Time Block Codes) system consists of a transmitter, a channel, and a receiver. The antenna diversity is exploited by using a communication system equipped with multiple antennas at the receiver [18]. The IEEE 802.11n standard exploits the use of MIMO systems to acquire throughputs as high as 600Mbps [19] and [20].

\section{B. Mimo-Wimax System}

MIMO is the use of multiple transmitters and receivers (multiple antennas) on both WiMAX base stations and subscriber devices to achieve improved performance in terms of bandwidth and distances. With MIMO, two simultaneous data streams can be sent, which doubles the bandwidth. It displays the Spectrum transmitter to antenna respectively.

The MIMAX is implemented with two channels and achieved with the same Bit Error Rate (BER) values for both the channels. The implementation of the MIMAX on WIMAX helps to take the integrated advantage of both the techniques MIMAX and WIMAX which mainly provides a supporting hand of the aim at covering a large geographical area of up to nearly 35 miles which is a very big advantage for the communicating vehicles of the vehicular network on the Intelligent Transportation System.

\section{SS-DSRC-MIMAX COMBIEND SYSTEM}

In this paper we proposed a noble approach of convergence 
of WiMax technology with vehicular DSRC system. Here in this work, we aim for Spread Spectrum based DSRC technology to be explored in MiMax network for future ITS. The block diagram of DSRC-WiMax system is shown in Fig. 2.We successfully simulated SS based DSRC system for image transmission. We also simulated WiMax system for data transmission. MIMO technology has been combined with WiMax technology for data transmission.

Spread Spectrum based DSRC, MIMO, WiMax technologies will explored separately in Matlab environment. Finally, the Spread Spectrum based DSRC system will be explored in MiMax network through Matlab simulation for ITS. (See Fig. 3, Fig. 4).

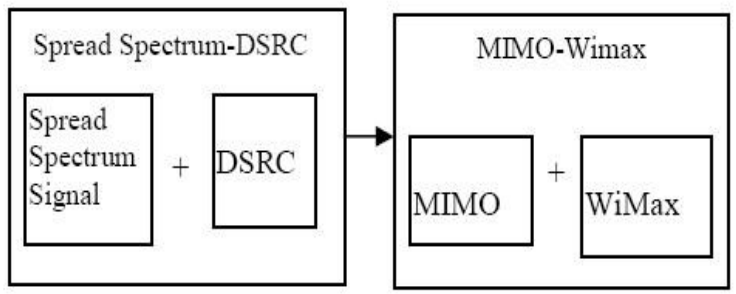

Fig. 2. Block diagram of SS-DSRC-MiMAX system.

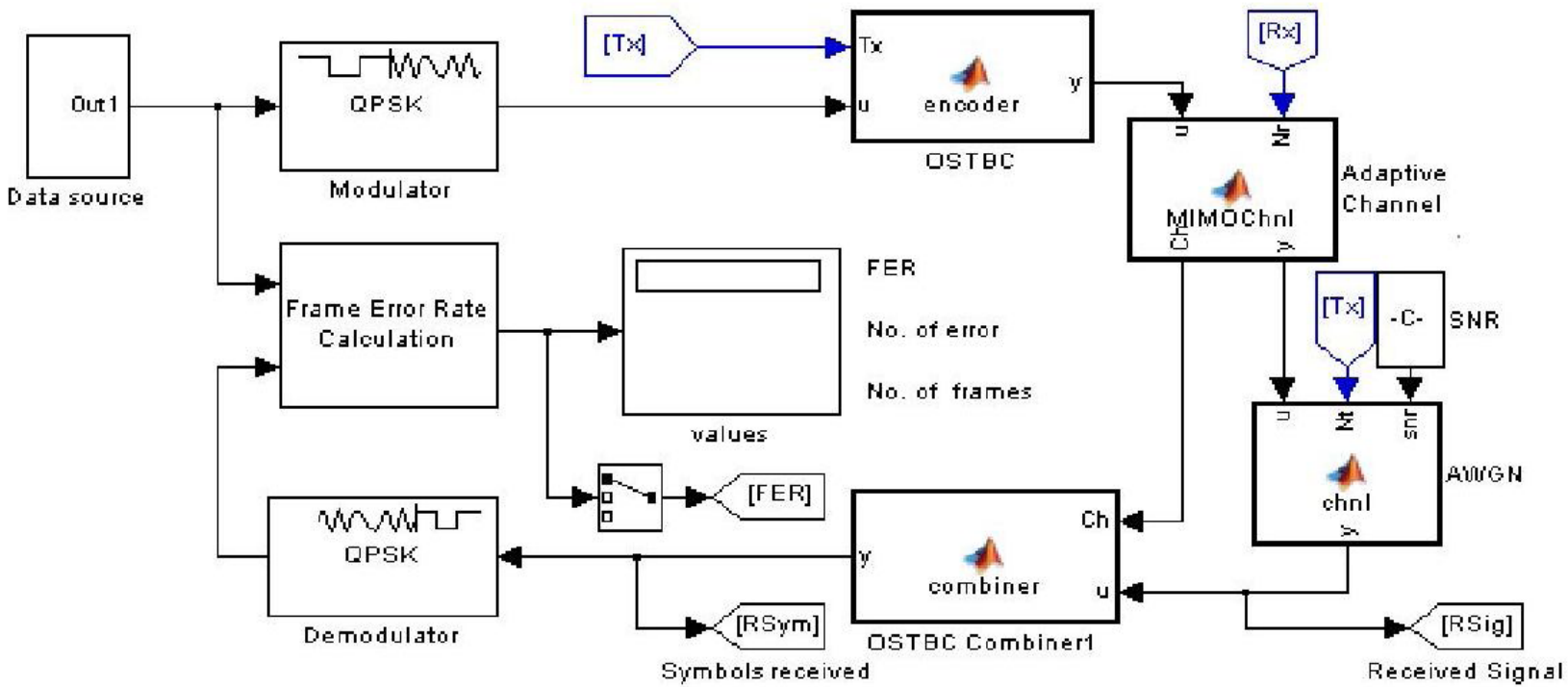

Fig. 3. MATLAB simulation model of STBC MIMO system.

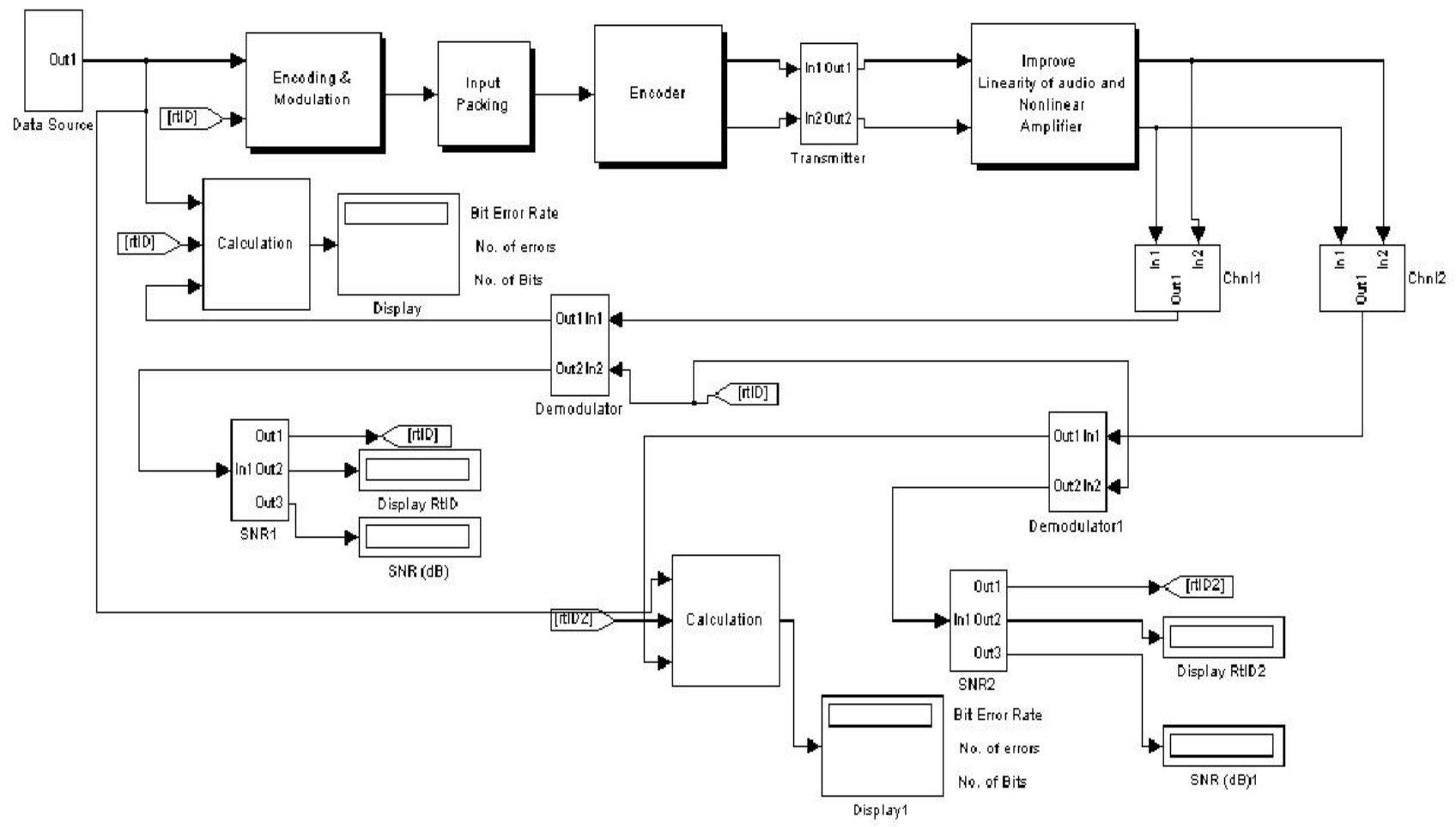

Fig. 4. MATLAB Simulation model of MIMax. 


\section{CONCUSSION}

The demanding needs of the ITS (Intelligent Transportation System) is fulfilled to a maximum extent. The coverage of data transmission is improved for a larger geographical area by converging the technologies of communication. Communication and RADAR technology will be converged in ITS using DSRC channels.

\section{REFERENCES}

[1] Accidents involving fatalities. [Online] Available: http://www.abs.gov.au/ausstats/abs@.nsf/Lookup/3875170E8478D1 71CA256CAE0016268F

[2] Fatal Heavy Vehicle Crashes Australia. Quarterly Bulletin (Octobe-December 2008). [Online] Available: http://www.infrastructure.gov.au/roads/safety/publications/2009/fhvc a_q42008.aspx

[3] Transport safety. Monash University Accident Research Centre.

[Online] Available: http://www.monash.edu.au/muarc/projects/its.html

[4] Mutiny.

[Online] Available: http://mutiny.wordpress.com/2007/02/19/road-accidents-in-india-cau sed-mostly-by-human-error/

[5] DW-World. [Online]

Available: http://www.dw-world.de/dw/article/0,,5519345,00.html

[6] S. Glisic and M. D. Katz, "Modeling of the code acquisition process for Rake receivers in CDMA wireless networks with multipath and transmitter diversity," IEEE J. Select. Areas Commun., vol. 19, pp. 21-32, Jan., 2001.

[7] K. H. Teo, Z. F. Tao, and J. Y. Zhang, "The mobile broadband WiMAX Standard [Standards in a Nutshell]," IEEE Signal Processing Magazine, vol. 24, pp. 144-148, 2007.

[8] K. Etemad, "Overview of mobile WiMAX technology and evolution," IEEE Communications Magazine, vol. 46, pp. 31-40, 2008.

[9] IEEE recommended practice for local and metropolitan area networks Coexistence of fixed broadband wireless access systems, IEEE Std 802.16.2-2004, 2004.

[10] W. C. Collier and R. J. Weiland, "Smart cars, smart highways," IEEE Spectrum, vol. 34, pp. 27-33, April 1994.

[11] WiMAX. [Online]. Available: http://www.wimax.com

[12] J. H. Winters, "On the capacity of radio communication systems with diversity in a Rayleigh fading environment," IEEE J. Select. Areas Commun., vol. SAC-5, pp. 871-878, June 1987.

[13] G. J. Foschini, "Layered space-time architecture for wireless communication in a fading environment when using multi-element antennas," Bell Labs Tech. J., pp. 41-59, 1996.

[14] G. J. Foschini and M. J. Gans, "On limits of wireless communications in a fading environment when using multiple antennas," Wireless Personal Commun., vol. 6, pp. 311-335, 1998.

[15] G. J. Foschini, G. D. Golden, R. A. Valenzuela, and P. W. Wolniansky, "Simplified processing for high spectral efficiency wireless communication employing multi-element arrays," IEEE J. Select. Areas Commun., vol. 17, pp. 1841-1852, Nov. 1999.

[16] Guide to securing WIMAX Wireless Communications, Karen Scarfone Cyrus Tibbs Matthew Sexton NIST Special Publication, pp. 800-127.

[17] V. Tarokh, N. Seshadri, and A. R. Calderbank, "Space-time codes for high data rate wireless communication: Performance criterion and code construction," IEEE Trans. Inform. Theory, vol. 44, no. 2, pp. 744-765, Mar. 1998

[18] E. G. Larsson and P. Stoica, "Space time block coding for wireless communications".

[19] IEEE P802.11n/D5.0, May 2008.
[20] H. Niu and Ngo, "Diversity and multiplexing switching in $802.11 \mathrm{n}$ MIMO systems," in Proc. of Fortieth Asilomar Conference on Signals, Systems and Computers, 2006. ACSSC'06., 2006, pp. 1644-1648.

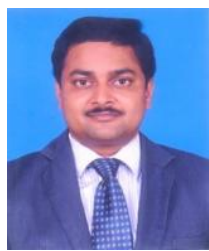

Debdatta Kandar was born in 1977 at Deulia, Kolaghat, Purba Medinipur, West Bengal, India. He received his Ph.D (Engg.) from Department of electronics and Telecommunication Engineering, Jadavpur University, Kolkata, West Bengal, India, in the year 2011. He also received his M.Tech from AAIDU and M.Sc.(Physics) from Vidyasagar University in the year 2006 and 2001 respectively. He has been awarded 'Young Scientist' award from Union Radio Science International (URSI GA 2005) at Vigyan Bhaban, Delhi, for his research work. President of India,

Dr. A. P. J. Abdul Kalam invited him at his residence on that occasion. Broadband Wireless Mobile Communication, Soft Computing and Radar Operation are the area of specializations. He is guiding several $\mathrm{PhD}$ and $\mathrm{ME}$ student in different universities. He has published more than 40 papers in different national and international conference and journals.

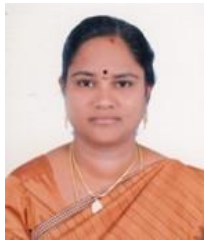

E. Bhuvaneswari was born in 1977, India. She received her M.E from Department of Computer Science and Engineering, Anna University, Chennai, Tamilnadu, India, in the year 2008. She also received her M.phil from Alagappa University in the year 2005 Data Mining and Soft Computing are the area of specializations. She has published 15 papers in different national and international conference.

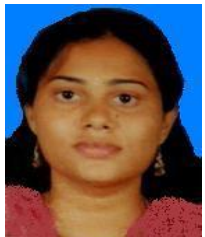

C. Auxilium Princy was born in 1983, at karaikal, India. She received her MCA from Department of Computer Science, Annamalai University, Tamilnadu, India, in the year 2007. She is doing her ME in SKP Engineering College, Thiruvannamalai, Tamilnadu. She presented National Conference On "Negotiation For Bridge Utilization Of HDPLIS in Bluetooth Scatternets".

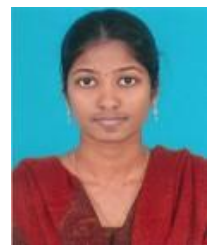

M. Nithya was born in 1990, India. She received her BE from Department of Information Technology, Anna University Chennai, Tamilnadu, India, in the year 2011 She doing her ME in SKP Engineering College, Thiruvannamalai,Tamilnadu. Database Technology and Soft Computing are the area of specializations.

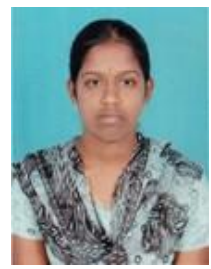

C. Subathradevi was born in 1990, India. She received her B.E from Department of Information Technology, Anna University Chennai, Tamilnadu, India, in the year 2011. She got her ME in SKP Engineering College, Thiruvannamalai, Tamilnadu. Network and Soft Computing are the area of specializations.

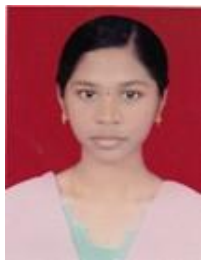

S. Porkodi was born in 1990, India. She received her B.E from Department of Computer Science and Engineering, Anna University Chennai, Tamilnadu, India, in the year 2011. She got her ME in SKP Engineering College, Thiruvannamalai, Tamilnadu. Network and Mobile Computing are the area of specializations. 